\title{
Structure of transverse momentum dependent (TMD) distributions at NNLO
}

\author{
Alexey Vladimirov* \\ Institut für Theoretische Physik, Universität Regensburg, D-93040 Regensburg, Germany \\ E-mail: Alexey.Vladimirovaphysik.uni-regensburg.de
}

We discuss the operator definition of the transverse momentum dependent parton distribution/fragmentation functions (TMDs). We present TMD operators in the completely (ultraviolet and rapidity) renormalized form suitable for straightforward evaluation of any matrix element. We show the consequences following from operator definition, such as renormalization group equations and small- $b_{T}$ operator product expansion. This methodology was successfully applied to unpolarized TMD distributions, where all perturbative quantities were calculated at NNLO. The obtained perturbative parts are important for any kind of phenomenology involving TMDs.

QCD Evolution 2016

May 30-June 03, 2016

National Institute for Subatomic Physics (Nikhef), Amsterdam

${ }^{*}$ Speaker. 


\section{Introduction}

The transverse momentum dependent parton distribution and fragmentation functions (TMDs) attract serious attention in the recent years. The TMDs grant the possibility to explore the structure of a hadron deeper in comparison to the integrated parton distributions. With the help of TMDs one gets access to the QCD dynamics hidden in multi-differential cross sections and to the tiniest details of the hadron spin structure. Many processes, such a Drell-Yan, Vector Boson/Higgs Production, Semi-Inclusive Deep Inelastic Scattering (SIDIS) and $e^{+} e^{-} \rightarrow 2$ hadrons, can be described in the terms of TMDs. All these processes are fundamental for current high energy colliders, like the LHC, KEK, SLAC, JLab or RHIC, and future planned facilities, like the EIC, AFTER@LHC, the $\mathrm{LHeC}$ or the ILC.

The general structure of TMD factorization theorems were first presented in the pioneering works of Collins and Soper [1]. However, only recently they were formulated in a rigorous quantum-field-theoretical form, and in the terms of individually well-defined TMDs [2, 3, 4, 5]. In this talk we discuss the properties and definition of only unpolarized TMDs, which have simplest structure among all TMDs. Nonetheless, the most essential points, such as divergence structure and interplay between perturbative and non-perturbative inputs can be studied in the unpolarized case and are universal for all TMDs.

The unpolarized TMDs have received much attention recently. The relevant factorization theorems have been explicitly checked at next-to-leading order (NLO), with various quantum numbers, by several groups (see e.g. $[4,6,7,8,9,10,11]$ ). However, there were no complete consideration TMDs within the TMD factorization formalism at NNLO. The main reason is that NNLO consideration requires accurate composition of all elements of the theory and calculus to obtain a meaningful result. Some next-to-next-to-leading order (NNLO) properties of these TMDs have been deduced from cross-section calculations made in collinear factorization framework, $[12,13])$. During the last year such study has been performed by two groups $[5,14,15]$ in different calculation schemes. This talk is based on $[5,14]$ as well as on results of further development.

\section{Definition of TMD operator}

The realization of the TMD factorization theorem at higher orders of perturbative QCD is not trivial. In fact, in the calculation one has to deal with several types of divergences (ultra-violet (UV), rapidity and infra-red (IR)). All these divergences have to be regularized and disentangled properly. Traditionally the discussion of the divergences structure is made on the level of the parton matrix elements. However, it is a bit misleading form. The point is that some divergences (namely, UV divergences, and rapidity divergences) are inherit part of TMD operator itself and not affected by the structure of a matrix element. Supplemented with proper renormalization factor a TMD operator is well-defined, process-independent and can be studied by any modern method of quantum field theory (in principal, including lattice simulations) without referring to factorization theorems.

The bare (unrenormalized and rapidity singular) quark TMD PDF operator is defined as fol- 
lowing

$$
O_{q}^{\text {bare }}\left(x, \vec{b}_{T}\right)=\frac{1}{2} \sum_{X} \int \frac{d \xi^{-}}{2 \pi} e^{-i x p^{+} \xi^{-}}\left\{T\left[\bar{q}_{i} \tilde{W}_{n}^{T}\right]_{a}\left(\frac{\xi}{2}\right)|X\rangle \gamma_{i j}^{+}\langle X| \bar{T}\left[\tilde{W}_{n}^{T \dagger} q_{j}\right]_{a}\left(-\frac{\xi}{2}\right)\right\},
$$

where $\xi=\left\{0^{+}, \xi^{-}, \vec{b}_{T}\right\}, n$ and $\bar{n}$ are light-cone vectors $\left(n^{2}=\bar{n}^{2}=0, n \cdot \bar{n}=1\right)$. The repeated color indices $a\left(a=1, \ldots, N_{c}\right.$ for quarks and $a=1, \ldots, N_{c}^{2}-1$ for gluons) are summed up. The Wilson lines $\tilde{W}_{n}^{T}(x)$ are rooted at the coordinate $x$ and continue to the light-cone infinity along the vector $n$, where it is connected by a transverse link to the transverse infinity (that is indicated by the superscript $T$ ). The gluon TMD PDF operator has the following form

$$
O_{g}^{\text {bare }}\left(x, \vec{b}_{T}\right)=\frac{1}{x p^{+}} \sum_{X} \int \frac{d \xi^{-}}{2 \pi} e^{-i x p^{+} \xi^{-}}\left\{T\left[F_{+\mu} \tilde{W}_{n}^{T}\right]_{a}\left(\frac{\xi}{2}\right)|X\rangle\langle X| \bar{T}\left[\tilde{W}_{n}^{T^{\dagger}} F_{+\mu}\right]_{a}\left(-\frac{\xi}{2}\right)\right\} .
$$

Here the Wilson lines are in the adjoint representation. The definition of the operators for the fragmentation functions follows a similar pattern, with the main difference that it should be evaluated on the final rather than initial states. The quark TMD FF operator is

$$
\begin{aligned}
\mathbb{O}_{q}^{\text {bare }}\left(z, \vec{b}_{T}\right)=\frac{1}{4 z N_{c}} \sum_{X} & \int \frac{d \xi^{-}}{2 \pi} e^{-i p^{+} \xi^{-} / z} \\
& \times\left\langle 0\left|T\left[\tilde{W}_{n}^{T \dagger} q_{j}\right]_{a}\left(\frac{\xi}{2}\right)\right| X, \frac{\delta}{\delta J}\right\rangle \gamma_{i j}^{+}\left\langle X, \frac{\delta}{\delta J}\left|\bar{T}\left[\bar{q}_{i} \tilde{W}_{n}^{T}\right]_{a}\left(-\frac{\xi}{2}\right)\right| 0\right\rangle,
\end{aligned}
$$

where $\delta / \delta J$ is to be understood as the state generated by the variation of the action with respect to the source $J$, which couples to external hadron fields. The anti-quark operator and gluon TMD FF operator can be found in [5]. In such language the similarity between TMDs are like the integrated parton densities is transparent. Basically, the only difference is the staple half-infinite path of the gauge link and singularities associated with it.

The hadron matrix elements of the bare TMD operators are the unsubtracted TMD distributions

$$
\begin{aligned}
\Phi_{f \leftarrow N}\left(x, \vec{b}_{T}\right) & =\left\langle N\left|O_{f}^{\text {bare }}\left(x, \vec{b}_{T}\right)\right| N\right\rangle, \\
\Delta_{f \rightarrow N}\left(z, \vec{b}_{T}\right) & =\left\langle\left. N\right|^{\dagger} \mathbb{O}_{f}^{\text {bare }}\left(z, \vec{b}_{T}\right) \mid N\right\rangle^{\dagger},
\end{aligned}
$$

where $N$ is a nucleon/hadron and the Hermitian conjugation of the states for TMDFFs indicates that these are final states to be placed inside the operator. Here variables $x / z$ acquire meaning of the momentum fraction carried by a parton from/into the hadron (it also explains the TMD labeling rule $f \leftarrow N$ or $t \rightarrow N$ ).

To complete the definition, TMD operator should be supplemented with UV and rapidity renormalization factors. We have

$$
\begin{aligned}
O_{f}\left(x, \vec{b}_{T}, \mu, \zeta\right) & =Z_{f}(\zeta, \mu) R_{f}(\zeta, \mu) O_{f}^{\text {bare }}\left(x, \vec{b}_{T}\right), \\
\mathbb{O}_{f}\left(z, \vec{b}_{T}, \mu, \zeta\right) & =Z_{f}(\zeta, \mu) R_{f}(\zeta, \mu) \mathbb{O}_{f}^{\text {bare }}\left(z, \vec{b}_{T}\right),
\end{aligned}
$$

where $Z_{f}\left(R_{f}\right)$ are the UV(rapidity) renormalization factor for TMD operators. The scales $\mu$ and $\zeta$ are the scales of UV and rapidity subtractions respectively. The explicit form of the factor $R$ is 
dictated by the TMD factorization theorem and reads

$$
R_{f}(\zeta, \mu)=\frac{\sqrt{S\left(\vec{b}_{T}\right)}}{\mathbf{Z b}},
$$

where the $S\left(\vec{b}_{T}\right)$ is the TMD soft factor, and $\mathbf{Z b}$ denotes the zero-bin contribution (the soft overlap of the collinear and soft sectors which appear in the factorization theorem) $[16,2,7,3]$.

The possibility to "just" renormalizes the rapidity divergences have been discussed in the TMD context for awhile [17]. Actually, in the TMD case this statement can be trivialized and reduced to the statement that the logarithm of TMD soft factor is linear in rapidity divergences, see e.g.[3, 18]. However, recent studies [19] demonstrate that renormalization properties of rapidity divergences is of a more fundamental nature. In particular, for system of parallel half infinite Wilson lines the rapidity renormalization is proved at all orders. This statement is also valid for the multi-parton scattering cross-section, what has been checked at two-loop order [20].

The form of the renormalization factor $R$ in the TMD case can be deduced from the soft factor. The soft factor is a vacuum expectation value of a certain configuration of Wilson lines, which depends on the process under investigation. For example, for SIDIS it reads

$$
\tilde{S}\left(\vec{b}_{T}\right)=\frac{\operatorname{Tr}_{c}}{N_{c}}\left\langle 0\left|T\left[S_{n}^{T \dagger} \tilde{S}_{\bar{n}}^{T}\right]\left(0^{+}, 0^{-}, \vec{b}_{T}\right) \bar{T}\left[\tilde{S}_{\bar{n}}^{T \dagger} S_{n}^{T}\right](0)\right| 0\right\rangle .
$$

The Wilson lines are defined as usual

$$
\begin{aligned}
& S_{n}(x)=P \exp \left[i g \int_{-\infty}^{0} d s n \cdot A(x+s n)\right], \\
& \tilde{S}_{\bar{n}}(x)=P \exp \left[-i g \int_{0}^{\infty} d s \bar{n} \cdot A(x+\bar{n} s)\right] .
\end{aligned}
$$

Note that collinear Wilson lines $W_{n}^{T}(x)$ used in TMD operators Eq. (2.2-2.3) are defined in the same way as soft Wilson lines $S_{n}^{T}(x)$. However, one should distinguish between $W$ and $S$, because they behave differently under regularization.

The explicit definition of zero-bin subtractions significantly depends on the rapidity regularization used. Thus, for a given regularization scheme it might be even impossible to define the zero-bin as a well-formed matrix element. Nonetheless, for any regularization scheme it has a very particular calculable expression. With a conveniently chosen rapidity regularization, the zero-bin subtractions are related to a particular combination of the soft factors [2]. Using the modified $\delta$ regularization [5], the zero-bin subtractions are literally equal to the soft factor: $\mathbf{Z b}=S\left(\vec{b}_{T}\right)$. It is not accidentally. In fact, the modified $\delta$-regularization scheme has been adapted such that this relation holds, which requires a different regularized form for collinear Wilson lines $W_{n(\bar{n})}(x)$ and for soft Wilson lines $S_{n(\bar{n})}(x)$. Therefore, in the modified $\delta$-regularization, the expression for the rapidity renormalization factor is

$$
\left.R^{f}(\zeta, \mu)\right|_{\delta \text {-reg. }}=\frac{1}{\sqrt{S\left(\vec{b}_{T} ; \zeta\right)}}
$$

The relation Eq. (2.12) was checked explicitly at NNLO in various kinematics in $[14,18,5]$. In the popular JCC regularization scheme by [2], the rapidity divergences are handled by tilting Wilson 
lines off-light cone by parameters $y$. The overlap region contribution has more cumbersome form, but nonetheless can be presented in the closed form as product of matrix elements of TMD soft factor. Then the renormalization factor also has the form (2.8)

$$
\left.R^{f}(\zeta, \mu)\right|_{J C C}=\sqrt{\frac{\tilde{S}\left(y_{n}, y_{c}\right)}{\tilde{S}\left(y_{c}, y_{\bar{n}}\right) \tilde{S}\left(y_{n}, y_{\bar{n}}\right)}} .
$$

The UV renormalization factors depend on the UV regularization method and the regularization scale $\mu$. In the similar fashion, the rapidity renormalization factors depend on the rapidity regularization method and the rapidity scale $\zeta$. Moreover, given that the soft factor is process independent (as argued with general arguments in $[21,2,3,4]$ and explicitly checked at NNLO in [18]), the "rapidity renormalization factors" are also process independent. Another important observation is that both UV and rapidity renormalization factors are the same for TMDPDF and TMDFF operators. This significantly simplifies the consideration of the operators and makes the whole approach more universal.

Now, given all previous considerations, we define the individual TMDs as

$$
\begin{aligned}
F_{f \leftarrow N}\left(x, \vec{b}_{T} ; \mu, \zeta\right) & =\left\langle N\left|O_{f}\left(x, \vec{b}_{T} ; \mu, \zeta\right)\right| N\right\rangle, \\
D_{f \rightarrow N}\left(z, \vec{b}_{T} ; \mu, \zeta\right) & =\left\langle\left. N\right|^{\dagger} \mathbb{O}_{f}\left(z, \vec{b}_{T} ; \mu, \zeta\right) \mid N\right\rangle^{\dagger} .
\end{aligned}
$$

Such a definition implies the following relation between bare and renormalized TMDs:

$$
\begin{aligned}
& F_{f \leftarrow N}\left(x, \vec{b}_{T} ; \mu, \zeta\right)=Z_{f}(\mu, \zeta) R_{f}(\mu, \zeta) \Phi_{f \leftarrow N}\left(x, \vec{b}_{T}\right), \\
& D_{f \rightarrow N}\left(z, \vec{b}_{T} ; \mu, \zeta\right)=Z_{f}(\mu, \zeta) R_{f}(\mu, \zeta) \Delta_{f \leftarrow N}\left(x, \vec{b}_{T}\right) .
\end{aligned}
$$

In fact, the same definition follows from the TMD factorization theorem, but in the presented formulation and operation strategy is clearer.

\section{Perturbatively calculable parts of TMD}

The TMDs, as a non-perturbative objects, are a highly involved functions. Any information on their behavior is important for phenomenological applications. The QCD perturbation theory can supply us the evolution equations and the small- $b_{T}$ matching of the TMDs on the corresponded integrated distributions. Naturally, both these inputs can be formulated on the operator language.

The renormalization group equations (RGEs) for TMDs are the result of the renormalization procedure. Since in the formulation $(2.6,2.7)$ have two renormalization constants, the scaling on the operator satisfies two equations. The UV RGE read

$$
\mu^{2} \frac{d}{d \mu^{2}} O_{f}\left(x, \vec{b}_{T}\right)=\frac{1}{2} \gamma^{f}(\mu, \zeta) O_{f}\left(x, \vec{b}_{T}\right), \quad \mu^{2} \frac{d}{d \mu^{2}} \mathbb{O}_{f}\left(z, \vec{b}_{T}\right)=\frac{1}{2} \gamma^{f}(\mu, \zeta) \mathbb{O}_{f}\left(z, \vec{b}_{T}\right) .
$$

and rapidity RGE (often called CSS equation) read

$$
\zeta \frac{d}{d \zeta} O_{f}\left(x, \vec{b}_{T}\right)=-\mathscr{D}^{f}\left(\mu, \vec{b}_{T}\right) O_{f}\left(x, \vec{b}_{T}\right), \quad \zeta \frac{d}{d \zeta} \mathbb{O}_{f}\left(z, \vec{b}_{T}\right)=-\mathscr{D}^{f}\left(\mu, \vec{b}_{T}\right) \mathbb{O}_{f}\left(z, \vec{b}_{T}\right) .
$$


The values of anomalous dimensions can be deduced from the renormalization constants. Using the standard RGE technique we obtain

$$
\gamma^{q}(\mu, \zeta)=2 \widehat{A D}\left(Z_{2}-Z_{q}\right), \quad \gamma^{g}(\mu, \zeta)=2 \widehat{A D}\left(Z_{3}-Z_{g}\right),
$$

where $\widehat{A D}$ represents the operator which extracts the anomalous dimension from the counterterm. The rapidity anomalous dimension $\mathscr{D}^{f}$ is [18]

$$
\mathscr{D}^{f}(\mu, \zeta)=-\left.\frac{d \ln R_{f}}{d \ln \zeta}\right|_{f \cdot p}=-\left.\frac{1}{2} \frac{d \ln R_{f}}{d \ln \delta^{+}}\right|_{f \cdot p},
$$

where $f$.p. denotes the extraction of the finite part, i.e. neglecting the poles in $\varepsilon$. The singular part of the factor $R$ is related to the renormalization factor as follows:

$$
\left.\frac{d \ln R_{f}}{d \ln \zeta}\right|_{\text {s.p. }}=\frac{d \ln Z_{f}}{d \ln \mu^{2}}
$$

where s.p. denotes the extraction of the singular part, i.e. the poles in $\varepsilon$. The last equation leads to the famous consistency relation for $(3.1,3.2)$

$$
\mu^{2} \frac{d}{d \mu^{2}}\left(-\mathscr{D}^{f}\left(\mu^{2}, \vec{b}_{T}\right)\right)=\zeta \frac{d}{d \zeta}\left(\frac{\gamma^{f}(\mu, \zeta)}{2}\right)=-\frac{\Gamma_{\text {cusp }}^{f}}{2}
$$

where $\Gamma$ is the cusp anomalous dimension. Due to the fact, that TMD operators in FF and PDF kinematics are renormalized by the same factors, the corresponding anomalous dimensions are also the same. The anomalous dimensions are independent of the regularization procedure.

Now let us turn to the regime of small- $b_{T}$. In this regime the TMD operator can be replaced by the first terms of small- $b_{T}$ Operator Product Expansion (OPE). The small- $b_{T}$ OPE is a formal operator relation, that relates operators with both light-like and space-like field separation to operators with only light-like field separation. It reads

$$
O\left(\vec{b}_{T}\right)=\sum_{n} C_{n}\left(\vec{b}_{T}, \mu_{b}\right) \otimes O_{n}\left(\mu_{b}\right)
$$

where $C_{n}$ are C-number coefficient functions, the $\mu_{b}$ is the scale of small- $b_{T}$ singularities factorization or the OPE matching scale. The operators on both sides of Eq. (3.7) are non-local along the same light-cone direction, but the operators $O_{n}$ are transversely local while $O\left(\vec{b}_{T}\right)$ is transversely non-local. Generally speaking, the operators $O_{n}$ are all possible operators with proper quantum numbers. They can be organized for instance according to twists. The scale parameter of such expansion $B_{T}$ has entirely non-perturbative nature. The twist expansion would also organize the $\mathrm{OPE}$ as a power series in $B_{T}^{-1}$

$$
C_{n}\left(\vec{b}_{T}, \mu_{b}\right) \sim\left(\frac{b_{T}}{B_{T}}\right)^{n} f\left(\ln \left(\vec{b}_{T}^{2} \mu_{b}^{2}\right)\right)
$$

where $f$ is some function. For $b_{T} \ll B_{T}$ it is reasonable to consider only the zeroth term of the OPE in Eq. (3.7), which gives the matching of the TMDs onto the integrated functions. The consideration of higher order terms is an interesting and a completely unexplored. 

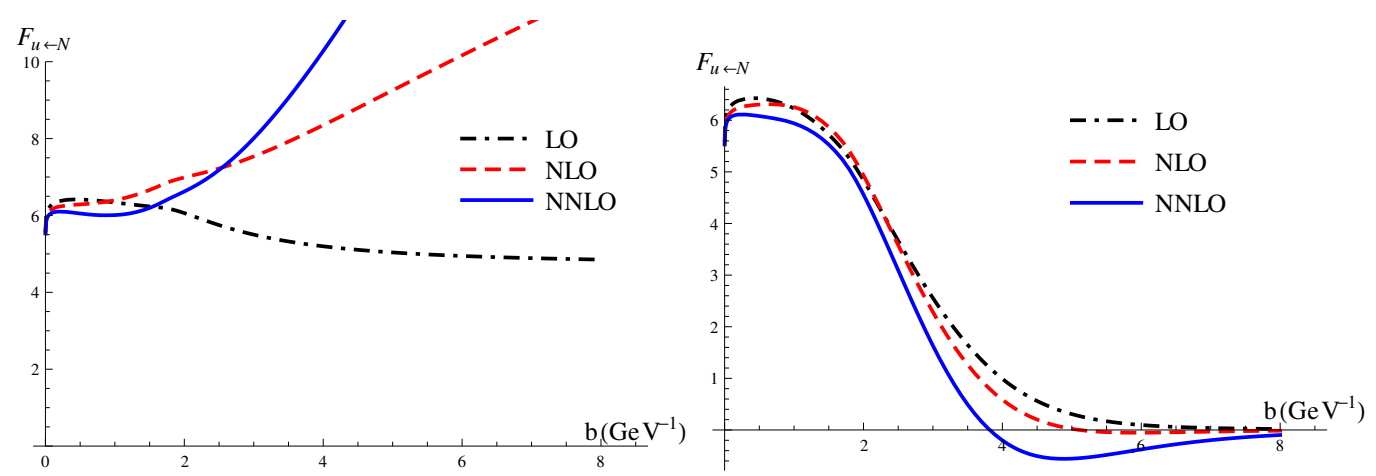

Figure 1: Comparison of the unpolarized TMD (u-)quark distribution at different orders of perturbation theory ( $x=0.1$ and $\mu_{b}=2 e^{-\gamma_{E}} / b^{*}\left(b_{\max }\right)$ with $b_{\max }=1.5$ ). (left) Pure perturbative expression as in (3.12). (right) The TMD with the non-perturbative contribution from the renormalon model [22].

For the TMDPDFs the leading order small- $b_{T}$ operator (i.e. the operator for the integrated PDF) is just a TMDPDF operator Eq. (2.2) at $b_{T}=0$, i.e.

$$
O_{f}^{\text {bare }}(x)=O_{f}^{\text {bare }}\left(x, \overrightarrow{0}_{T}\right)
$$

while for FF kinematics one has an extra normalization factor

$$
\mathbb{O}_{f}^{\text {bare }}(z)=z^{2-2 \varepsilon} \mathbb{O}_{f}^{\text {bare }}\left(z, \overrightarrow{0}_{T}\right) .
$$

In this way the leading terms of the OPEs at small $b_{T}$ read

$$
\begin{aligned}
& O_{f}\left(x, \vec{b}_{T} ; \mu, \zeta\right)=\sum_{f^{\prime}} C_{f \leftarrow f^{\prime}}\left(x, \vec{b}_{T} ; \mu, \zeta, \mu_{b}\right) \otimes O_{f^{\prime}}\left(x, \mu_{b}\right)+\mathscr{O}\left(\frac{b_{T}}{B_{T}}\right), \\
& \mathbb{O}_{f}\left(z, \vec{b}_{T} ; \mu, \zeta\right)=\sum_{f^{\prime}} \mathbb{C}_{f \rightarrow f^{\prime}}\left(z, \vec{b}_{T} ; \mu, \zeta, \mu_{b}\right) \otimes \frac{\mathbb{O}_{f^{\prime}}\left(z, \mu_{b}\right)}{z^{2-2 \varepsilon}}+\mathscr{O}\left(\frac{b_{T}}{B_{T}}\right),
\end{aligned}
$$

where the symbol $\otimes$ is the Mellin convolution in variable $x$ or $z$, and $f, f^{\prime}$ enumerate the various flavors of partons. The running on the scales $\mu, \mu_{b}$ and $\zeta$ is independent of the regularization scheme and it is dictated by the renormalization group equations. Taking the hadron matrix elements of the operators we obtain the small- $b_{T}$ matching of the TMDs to corresponding integrated functions,

$$
\begin{aligned}
& F_{f \leftarrow N}\left(x, \vec{b}_{T} ; \mu, \zeta\right)=\sum_{f^{\prime}} C_{f \leftarrow f^{\prime}}\left(x, \vec{b}_{T} ; \mu, \zeta, \mu_{b}\right) \otimes f_{f^{\prime} \leftarrow N}\left(x, \mu_{b}\right)+\mathscr{O}\left(\frac{b_{T}}{B_{T}}\right), \\
& D_{f \rightarrow N}\left(z, \vec{b}_{T} ; \mu, \zeta\right)=\sum_{f^{\prime}} \mathbb{C}_{f \rightarrow f^{\prime}}\left(z, \vec{b}_{T} ; \mu, \zeta, \mu_{b}\right) \otimes \frac{d_{f^{\prime} \rightarrow N}\left(z, \mu_{b}\right)}{z^{2-2 \varepsilon}}+\mathscr{O}\left(\frac{b_{T}}{B_{T}}\right) .
\end{aligned}
$$

The integrated functions (PDFs and FFs) depend only on the Bjorken variables ( $x$ for PDFs and $z$ for FFs) and renormalization scale $\mu$, while all the dependence on the transverse coordinate $b_{T}$ and rapidity scale is contained in the matching coefficient and can be calculated perturbatively.

In order to calculate the leading matching coefficients of the OPE, we perform the calculation of TMD distributions on parton targets. Consequently comparing left- and right-hand sides of 

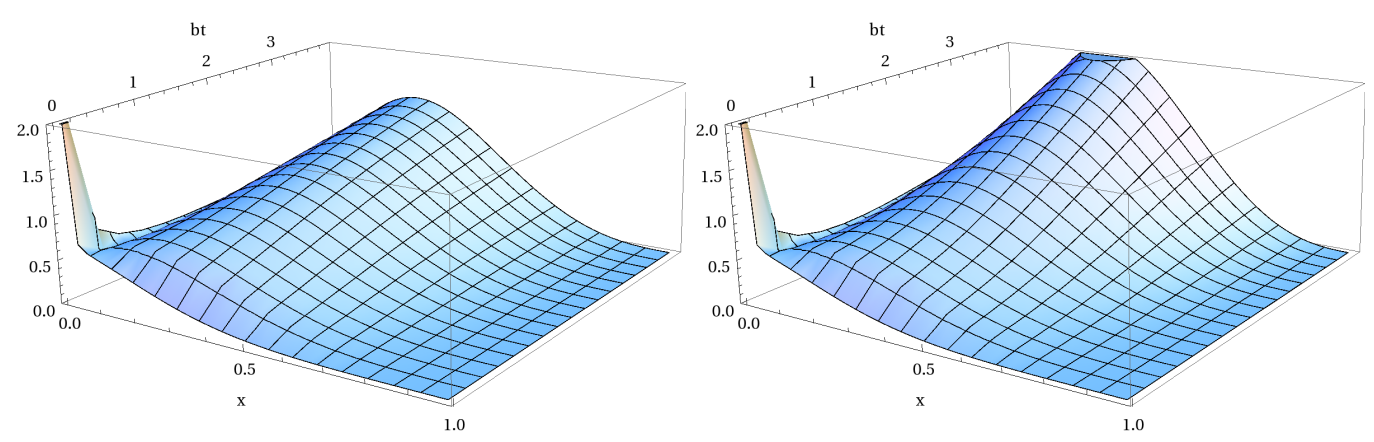

Figure 2: The shape of the perturbative part (3.12) of the unpolarized TMD (u-)quark distribution (multiplied by $x$ ) at different orders of perturbation theory $\left(\mu_{b}=2 e^{-\gamma_{E}} / b^{*}\left(b_{\max }\right)\right.$ with $b_{\max }=1.5$ ), at NLO (left) and at NNLO (right).

(3.12) we obtain the perturbative expansion for matching coefficients from the partonic matrix elements. For example at NNLO we have

$$
\begin{aligned}
& C_{f \leftarrow f^{\prime}}^{[2]}=F_{f \leftarrow f^{\prime}}^{[2]}-\sum_{r} C_{f \leftarrow r}^{[1]} \otimes f_{r \leftarrow f^{\prime}}^{[1]}-f_{f \leftarrow f^{\prime}}^{[2]}, \\
& \mathbb{C}_{f^{\prime} \rightarrow f}^{[2]}=D_{f^{\prime} \rightarrow f}^{[2]}-\sum_{r} \mathbb{C}_{f \rightarrow r}^{[1]} \otimes \frac{d_{r \rightarrow f^{\prime}}^{[1]}}{z^{2-2 \varepsilon}}-\frac{d_{f^{\prime} \rightarrow f}^{[2]}}{z^{2-2 \varepsilon}} .
\end{aligned}
$$

The matching procedure ensures the cancelation of the IR divergences in the matching coefficients. In $\delta$-regularization scheme these divergences are regularized by the dimensional regularization.

In the paper [5] all matching coefficients (including PDF and FF kinematic, quark, anti-quark and gluon operators and their mixing) are calculated at the NNLO order. The results for the TMDPDFs agrees with the results of [13], where they have been obtained by explicit factorization of cross-section for Drell-Yan process. The results for quark TMDFFs were partially presented in [14], while all the rest expression (including also the gluon TMDFFs at NLO) are novel. Also in [5] one can find the collection of all normalization factors for TMD operator up to NNLO order. These anomalous dimensions and matching coefficients are necessary for accurate phenomenological studies, and allow to consider exclusive and inclusive processes on the same level of theoretical accuracy. The comparison of different orders is presented in fig.1 and fig.2.

\section{Discussion}

The present understanding of the perturbative methodology for TMD operator is comprehensive. The structure of singularities (rapidity and UV) is well understood, and the necessary renormalization theorems are proven [19]. It is also clear how to construct the small- $b_{T}$ OPE. These finding has been checked in the unpolarized case up to NNLO. The next step of the perturbative applications is polarized TMD distributions. Some of polarized distributions, e.g. helicity and transvesity TMD distributions, were studied previously in the perturbative regime [9, 10]. However these studies were not systematic, and the most part of TMDs is completely untouched. With 
the present understanding of TMD factorization a systematic study of any TMD distribution can be done. Especially interesting is to consider the TMDs that match to the twist-three operators at the leading order of OPE (e.g. Sivers function).

Another side of the TMD study is the non-perturbative contributions. The understanding of the non-perturbative part should be considered as a primal goal, since it allows to look into the deep of the hadron structure. One of the significant problem is to clearly separate the perturbative and non-perturbative parts. This procedure is not well formulated at the moment. But definitely there are several important ingredients. First, the perturbative part should be known very precise. With the current NNLO results (at least for unpolarized distributions) we are much close to this limit. Second, we should find a distinctive feature of non-perturbative part that would allow to split the contributions. But even in this cases one cannot guaranty the clear and unique separation.

Additional problem is the several sources of the non-perturbative correction to TMD. Namely, the rapidity anomalous dimension has a non-perturbative contribution, and the higher small- $b_{T}$ OPE terms also present collective source of a non-perturbative contribution. The disentanglement of these sources is theoretically clear, but phenomenologically not even considered. Essential that rapidity anomalous dimension is $x / z$-independent, while the higher-orders of OPE are naturally dependent. Moreover the leading order $x$-dependence can be calculated, e.g. in the renormalon scheme [22].

The obtained NNLO coefficients are necessary in order to pursue phenomenological studies at $\mathrm{N}^{3} \mathrm{LL}$ accuracy. Some recent developments towards this goal can be found in $[23,19]$. It opens the door to a very precise estimate of these perturbatively calculable contributions. The phenomenological applications of these results will be exploited in future works. We expect all these efforts to be necessary in order to have a unified picture of Drell-Yan, semi-inclusive DIS and $e^{+} e^{-} \rightarrow 2$ hadrons.

\section{References}

[1] J. C. Collins and D. E. Soper, Nucl. Phys. B 193 (1981) 381 [Nucl. Phys. B 213 (1983) 545].

[2] J. C. Collins, Foundations of perturbative QCD (Cambridge University Press, Cambridge, 2011).

[3] M. G. Echevarria, A. Idilbi and I. Scimemi, Phys. Lett. B 726 (2013) 795 [arXiv:1211.1947 [hep-ph]].

[4] M. G. Echevarria, A. Idilbi and I. Scimemi, Phys. Rev. D 90 (2014) 1, 014003 [arXiv:1402.0869 [hep-ph]].

[5] M. G. Echevarria, I. Scimemi and A. Vladimirov, doi:10.1007/JHEP09(2016)004 arXiv:1604.07869 [hep-ph].

[6] S. M. Aybat and T. C. Rogers, Phys. Rev. D 83 (2011) 114042 [arXiv:1101.5057 [hep-ph]].

[7] M. G. Echevarria, A. Idilbi and I. Scimemi, JHEP 1207 (2012) 002 [arXiv:1111.4996 [hep-ph]].

[8] A. A. Vladimirov, JHEP 1408, 089 (2014) [arXiv:1402.3182 [hep-ph]].

[9] A. Bacchetta and A. Prokudin, Nucl. Phys. B 875 (2013) 536 [arXiv:1303.2129 [hep-ph]].

[10] M. G. Echevarria, T. Kasemets, P. J. Mulders and C. Pisano, JHEP 1507 (2015) 158 [arXiv:1502.05354 [hep-ph]].

[11] R. Zhu, P. Sun and F. Yuan, Phys. Lett. B 727 (2013) 474 [arXiv:1309.0780 [hep-ph]]. 
[12] S. Catani and M. Grazzini, Eur. Phys. J. C 72 (2012) 2013 [Eur. Phys. J. C 72 (2012) 2132] [arXiv:1106.4652 [hep-ph]].

[13] T. Gehrmann, T. Lubbert and L. L. Yang, Phys. Rev. Lett. 109 (2012) 242003 [arXiv:1209.0682 [hep-ph]].

[14] M. G. Echevarria, I. Scimemi and A. Vladimirov, Phys. Rev. D 93 (2016) 1, 011502 [arXiv:1509.06392 [hep-ph]].

[15] T. Lübbert, J. Oredsson and M. Stahlhofen, JHEP 1603 (2016) 168 doi:10.1007/JHEP03(2016)168 [arXiv:1602.01829 [hep-ph]].

[16] A. V. Manohar and I. W. Stewart, Phys. Rev. D 76 (2007) 074002 [hep-ph/0605001].

[17] J. Y. Chiu, A. Jain, D. Neill and I. Z. Rothstein, JHEP 1205 (2012) 084 doi:10.1007/JHEP05(2012)084 [arXiv:1202.0814 [hep-ph]].

[18] M. G. Echevarria, I. Scimemi and A. Vladimirov, Phys. Rev. D 93, no. 5, 054004 (2016) [arXiv:1511.05590 [hep-ph]].

[19] A. A. Vladimirov, arXiv:1610.05791 [hep-ph].

[20] A. Vladimirov, arXiv:1608.04920 [hep-ph].

[21] J. C. Collins and A. Metz, Phys. Rev. Lett. 93 (2004) 252001 [hep-ph/0408249].

[22] I. Scimemi and A. Vladimirov, arXiv:1609.06047 [hep-ph].

[23] Y. Li and H. X. Zhu, [arXiv:1604.01404 [hep-ph]]. 